University of Nebraska - Lincoln

DigitalCommons@University of Nebraska - Lincoln

April 2000

\title{
The interplay between the surface band structure and possible surface reconstructions of $\mathrm{Mo}(112)$
}

\author{
T. McAvoy \\ University of Nebraska-Lincoln \\ Jun Zhang \\ University of Nebraska-Lincoln, jzhang5@unl.edu \\ C. Waldfried \\ University of Nebraska-Lincoln \\ D.N. Mcllroy \\ University of Nebraska-Lincoln \\ Peter A. Dowben \\ University of Nebraska-Lincoln, pdowben@unl.edu \\ See next page for additional authors
}

Follow this and additional works at: https://digitalcommons.unl.edu/physicsdowben

Part of the Physics Commons

McAvoy, T.; Zhang, Jun; Waldfried, C.; Mcllroy, D.N.; Dowben, Peter A.; Zeybek, O.; Bertrams, T.; and Barrett, S.D., "The interplay between the surface band structure and possible surface reconstructions of $\mathrm{Mo}(112) "$ (2000). Peter Dowben Publications. 118.

https://digitalcommons.unl.edu/physicsdowben/118

This Article is brought to you for free and open access by the Research Papers in Physics and Astronomy at DigitalCommons@University of Nebraska - Lincoln. It has been accepted for inclusion in Peter Dowben Publications by an authorized administrator of DigitalCommons@University of Nebraska - Lincoln. 


\section{Authors}

T. McAvoy, Jun Zhang, C. Waldfried, D.N. Mcllroy, Peter A. Dowben, O. Zeybek, T. Bertrams, and S.D. Barrett 


\title{
The interplay between the surface band structure and possible surface reconstructions of $\mathrm{Mo}(112)$
}

\author{
T. McAvoy 1,a, J. Zhang 1,b, C. Waldfried ${ }^{1, \mathrm{c}}$, D.N. McIlroy ${ }^{1, \mathrm{~d}}$, P.A. Dowben ${ }^{1, \mathrm{e}}$, O. Zeybek ${ }^{2}$, T. Bertrams ${ }^{2}$, \\ and S.D. Barrett ${ }^{3}$ \\ 1 Department of Physics and Astronomy, and the Center for Materials Research and Analysis, University of Nebraska-Lincoln, \\ Lincoln, Nebraska 68588-0111, USA \\ 2 Surface Science Research Center, University of Liverpool, Liverpool L69-3BX, UK \\ 3 Surface Science Research Center and Department of Physics, University of Liverpool, Liverpool L69-3BX, UK
}

Submitted March 1999; revised October 1999

\begin{abstract}
The experimental band structure of $\mathrm{Mo}(112)$ and the effects by temperature and adsorbate are presented. A surface resonance, identified as crossing the Fermi level at about $1 / 3$ from $\bar{\Gamma}$ to $\bar{X}$ of surface Brillouin zone, was observed to be very sensitive to both contamination and temperature. We find evidence of adsorbate and temperature induced reconstruction of the Mo(112) surface. Examination of low-energy electron diffraction (LEED) and scanning tunneling microscopy (STM) data provides evidence for an adsorbate induced reconstruction of the $\mathrm{Mo}(112)$ surface with periodicities consistent with the Fermi level crossing of the surface resonance. The reconstruction is found to occur at coverages as low as 0.03 Langmuirs of oxygen or carbon. The reconstruction and/or adsorbate affects the density of states and bands near the Fermi level of $a_{1}$ symmetry.
\end{abstract}

Keywords: X-ray diffraction and scattering, Solid surfaces and solid-solid interfaces, Surface and interface electron states

\section{Introduction}

A surface reconstruction can be a result of an effort to lower the total surface free energy $[1,2]$. Such reconstructions of the (100) surface of both molybdenum and tungsten have been studied extensively [1,3], providing classic examples of the various reconstruction driving mechanisms. A change in the surface lattice that accompanies a change in the surface band structure, leading to reduction of the density of states at the Fermi energy, particularly for a surface state, can drive a surface reconstruction. This coupled lattice distortion and change in electronic structure can have a dependence on temperature and adsorbate coverage.

Studies of the (100) surface of both molybdenum and tungsten have been prevalent [3-8], we hope to provide a more complete understanding of surface recon-

\footnotetext{
${ }^{a}$ Innovative Micro Technology, Santa Barbara, California.

${ }^{\mathrm{b}}$ Department of Physics, Florida International University, University Park, Miami, FL 33199, USA

${ }^{\mathrm{c}}$ Eaton SEO, 7600 Standish Place, Rockville, Maryland 20855-2798, USA

${ }^{\mathrm{d}}$ Department of Physics, Engineering and Physics Bldg., University of Idaho Moscow, ID 83844-0903, USA

e E-mail: pdowben@unl.edu
}

structions by studying the (112) surface of molybdenum. There have been numerous studies of adsorbates on Mo(112) [9-12] and on Mo(111) [13]. A number of adsorbates are seen to reconstruct the $\operatorname{Mo}(111)$ [13] and W(111) [13-15] surfaces to facets of (112) orientation. For this reason, the (112) surface is important as it represents a surface with either great stability or a low density of states near the Fermi level as this is the end orientation in these faceting studies. In fact the $\operatorname{Mo}(112)$ surface is an open surface that also exhibits surface reconstructions.

Here, we present the experimental band structure of $\mathrm{Mo}(112)$ and show that there is some agreement between theory and experiment for the surface resonance that crosses the Fermi level along the $\langle\overline{1} 11\rangle$ direction $(\bar{\Gamma}-\bar{X})[16,17]$. We show that the band structure is temperature dependent below $250 \mathrm{~K}$. Above $250 \mathrm{~K}$ the photoemission intensities are consistent with the dynamic motions of surface atoms as a function of temperature, as outlined elsewhere [18]. An understanding of the stability molybdenum surfaces, in terms of the electronic structure, may have more general significance beyond the general tendency of Cr, Mo and W to reconstruct [2]. In this sense, this study complements the experimental and theoretical band structure studies of $\operatorname{Mo}(100)$ and $\operatorname{Mo}(110)[1,8,19-22]$. 


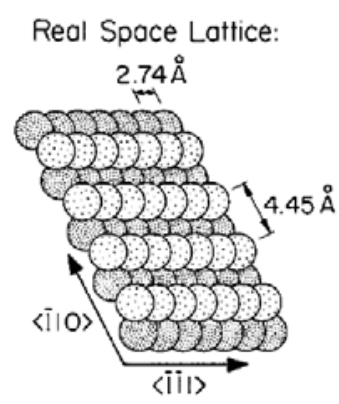

Surface Brillouin Zone:

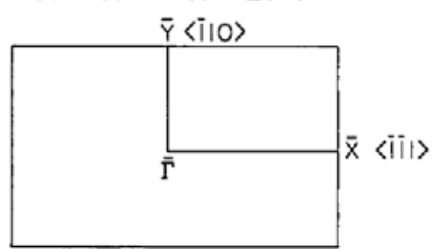

Fig. 1. A schematic of the rectangular surface structure of unreconstructed $\operatorname{Mo}(112)$ (at top) and the surface Brillouin zone and critical points labeled (at bottom).

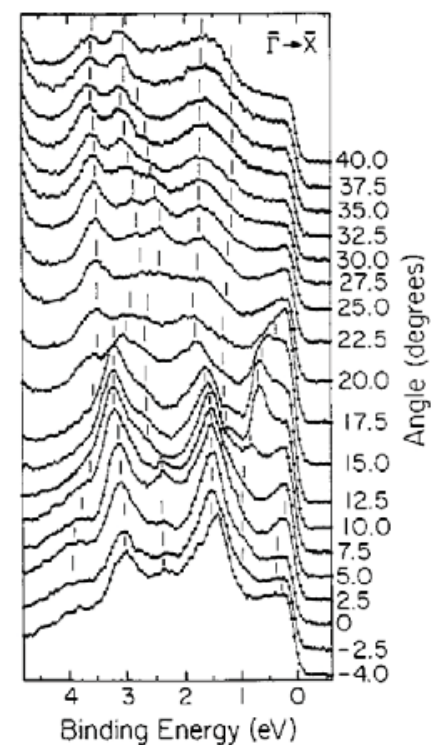

Fig. 2. The photoemission spectra as a function of emission angle along $\bar{\Gamma}$ to $\bar{X}$ are shown for clean Mo(112). The photon energy is 18 $\mathrm{eV}$ and the light incidence angle is $45^{\circ}$.

\section{Experimental}

The inverse photoemission (IPES) and low energy electron diffraction (LEED) experiments were carried out in a UHV chamber with a base pressure better than $1 \times 10^{-10}$ torr. The IPES system, with a resolution of $420 \mathrm{meV}$, incorporates an Erdman-Zipf electron gun [23] along with a Geiger-Müller detector with a $\mathrm{SrF}_{2}$ window in an apparatus previously described elsewhere [24]. The electron energy was swept from 5.5 to $12.5 \mathrm{eV}$, at incident angles between normal and 60 degrees, or from zone center $(\bar{\Gamma})$

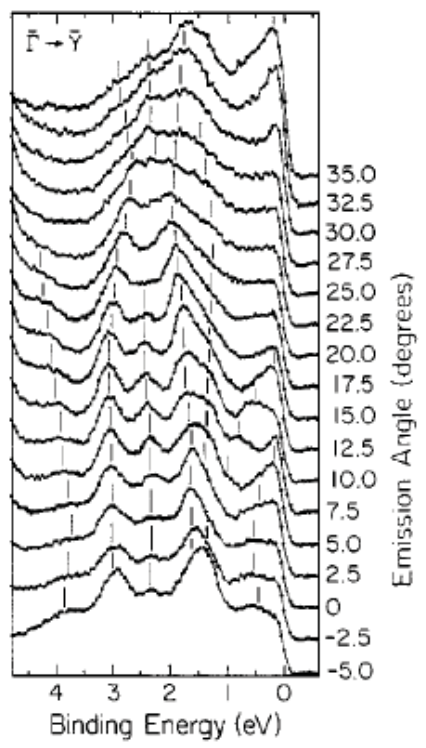

Fig. 3. The photoemission spectra as a function of emission angle along $\bar{\Gamma}$ to $\bar{Y}$ are shown for clean $\operatorname{Mo}(112)$. The photon energy is 18 $\mathrm{eV}$ and the light incidence angle is $45^{\circ}$.

out to the Brillouin zone edge $(\bar{X})$. The photoemission (PES) experiments, with a resolution between 0.10 and $0.15 \mathrm{eV}$, were carried out at the Synchrotron Radiation Center in Stoughton, Wisconsin in a UHV chamber employing a hemispherical electron energy analyzer with an angular acceptance of $\pm 1^{\circ}$, which has been described elsewhere [25]. The photoelectrons were collected with emission angles defined with respect to the surface normal.

The order of the Mo(112) surface was verified by LEED and the absence of surface contamination by photoemission and the sample was prepared using well-established procedures [18]. The surface of the $\mathrm{Mo}(112)$ crystal was cleaned by repeated annealing in oxygen and electron bombardment (flashing) and the crystal temperature was monitored with a W-5\%Re W-26\%Re thermocouple with an accuracy of $5 \mathrm{~K}$. Exposure of the Mo(112) crystal to oxygen was controlled with the use of a standard UHV leak valve.

The LEED studies were complemented by STM experiments which were carried out with a Omicron room temperature UHV STM at the Surface Science Research Center in Liverpool, UK. All measurements were performed in the constant current mode at a base pressure of $1.0 \times 10^{-10}$ torr.

\section{Band structure near the Fermi level}

A large sequence of angle resolved photoemission spectra were taken along the high symmetry directions of Mo(112), schematically shown in Figure 1, for different photon energies (18, 50 and $55 \mathrm{eV}$ ). Shown in Figures 2 and 3 are the emission angle photoemission spectra along 


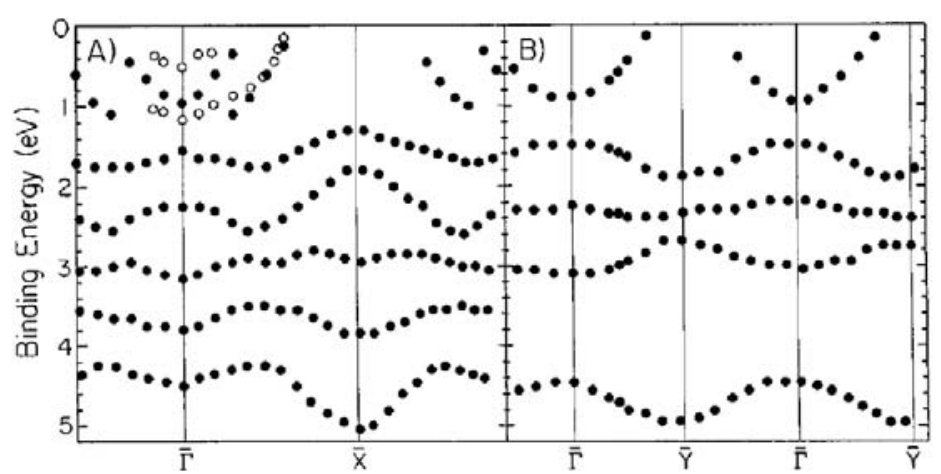

Fig. 4. The experimental band structure compiled from photoemission spectra taken at a photon energy of $50 \mathrm{eV}$. The band structure along $\Gamma^{-}$to $X^{-}$ and into the second zone is shown at left (A) and the band structure along $\Gamma^{-}$to $Y^{-}$and plotted even further into the second zone is shown at right (B). For comparison, the experimental bands near the Fermi level have been plotted for $18 \mathrm{eV}$ photon energy with the open symbols along $\Gamma^{-}$to $X^{-}$.

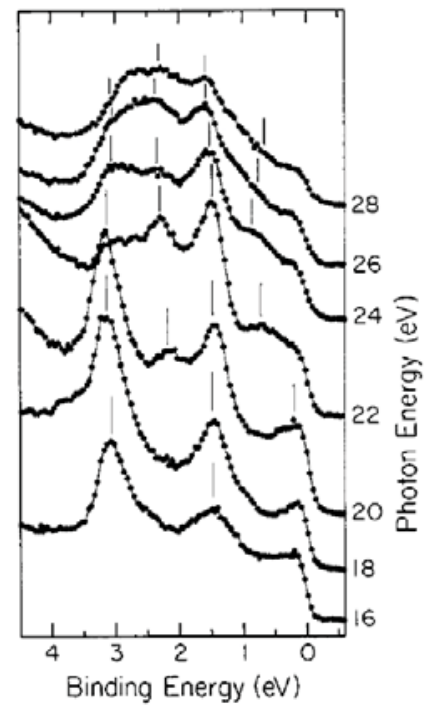

Fig. 5. The photon energy dependence of the energy distribution curves. The photoemission spectra are taken at normal emission for $p$ polarized light (light incidence angle of 70 degrees).

$\bar{\Gamma}-\bar{X}$ and $\bar{\Gamma}-\bar{Y}$ respectively. From these, and like data, an experimental band structure has been constructed, with the binding energies plotted against the component of wave vector parallel with the surface determined according to:

$$
\mathbf{k}_{\|}=\left\{\left(2 m E_{\mathrm{kin}} / \hbar^{2}\right)\right\}^{1 / 2} \sin \theta
$$

where, for IPES, $E_{\text {kin }}$ is the kinetic energy of the incident electrons and $\theta$ is the incidence angle relative to normal incidence and, for PES, $E_{\mathrm{kin}}$ is the kinetic energy of the emitted photoelectron and $\theta$ is the emission angle relative to the surface normal. This is plotted out in Figure 4, for one photon energy $(50 \mathrm{eV})$ where the band structure can be plotted for several surface Brillouin zones as shown.

The binding energies of the bulk bands vary with photon energy. The dependence of the bands upon photon energy, as seen in Figure 5, significantly affects all the bands except those bands at approximately $3.1 \mathrm{eV}$ and $1.5 \mathrm{eV}$ binding energy. Since the photon energy dependent spectra are taken for normal emission or $\mathbf{k}_{\|}=\mathbf{0}$, in Figure 5, the bands exhibiting photon energy dependence are dispersing with $\mathbf{k}_{\perp}$ and are therefore bulk bands. The states at about $4.3 \mathrm{eV}, 2.4 \mathrm{eV}$, and $0.7 \mathrm{eV}$ (the latter ranging from $0.3 \mathrm{eV}$ to $1.0 \mathrm{eV}$ depending upon photon energy) are clearly bulk bands. In Figure 5, the critical point of the bulk band structure, along the surface normal is at about $20 \pm 1 \mathrm{eV}$.

When the binding energies do not change with photon energy (no dependence upon the wave vector normal to the surface or $\mathbf{k}_{\perp}$ ) this indicates conservation of two dimensionality of state and suggests surface sensitivity. The band crossing of Fermi level at about midway along $\bar{\Gamma}-\bar{X}$ is largely unaffected by photon energy, as one approaches the Fermi level crossing, as indicated in Figure 4. The fact that the states at approximately $3.1 \mathrm{eV}$ and $1.5 \mathrm{eV}$ binding energy and the Fermi level crossing at about midway along $\bar{\Gamma}-\bar{X}$ are affected by small amounts of contamination provides further indication that these band have surface weight. None of the bands exhibiting surface sensitivity or conservation of two dimensionality of state appear to fall in a gap of the calculated bulk band structure $[16,17]$ and are therefore surface resonances rather than surface states. There is agreement between the experimental surface resonance crossing $E_{\mathrm{F}}$ at about midway along $\bar{\Gamma}-\bar{X}$ and a calculated surface resonance with a Fermi level crossing at about the same position along $\bar{\Gamma}-\bar{X}$ [16]. It should be mentioned that the state at $0.7 \mathrm{eV}$ (the latter ranging from $0.3 \mathrm{eV}$ to $1.0 \mathrm{eV}$ depending upon photon energy), may also have some surface character as indicated by the sensitivity of this state to contamination as discussed below.

Combining both PES and IPES data there is strong evidence for a Fermi level crossing for the surface resonance along $\bar{\Gamma}$ to $\bar{X}$. In Figure 6, we have plotted the experimental band structure for this one state using both photoemission and inverse photoemission results, with the surface resonances, from the calculated band struc- 


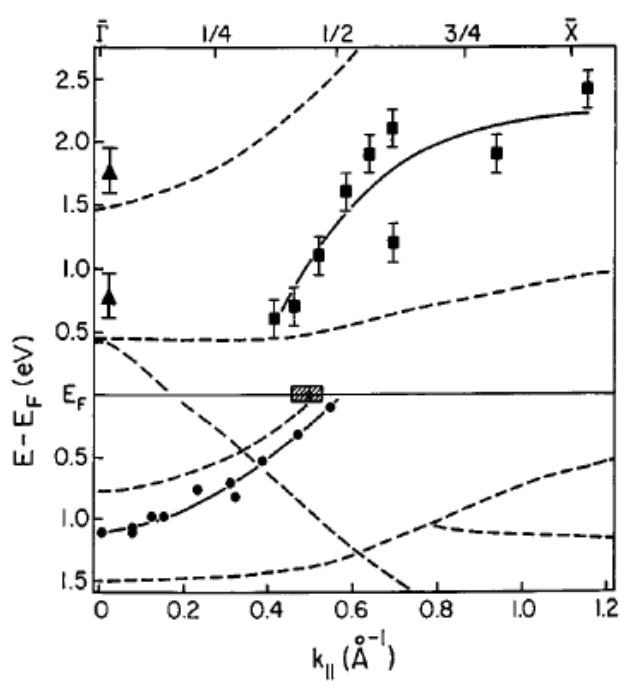

Fig. 6. Experimental Mo(112) band structure constructed from both angle-resolved photoemission $(h v=18$ and $50 \mathrm{eV}$ ) and inverse photoemission $\left(E_{\mathrm{i}}=5.5 \rightarrow 12.5 \mathrm{eV}\right)$ for the surface resonance/surface states along $\bar{\Gamma}$ to $\bar{X}$. The position of Fermi level crossing, of the state with considerable surface weight, has been plotted from the density of states (see text) as a box at $E_{\mathrm{F}}$. For comparison, the calculated surface resonances have been plotted from reference [16], using the dashed lines.

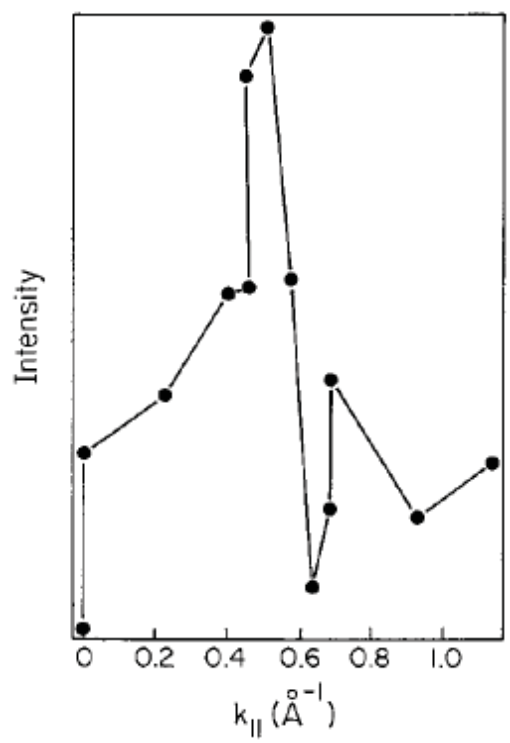

Fig. 7. The intensity in inverse photoemission, at the Fermi level, as a function of wave vector $k_{\|}$has been plotted along $\bar{\Gamma}$ to $\bar{X}$.

ture of Yakovkin [16], for comparison. On the photoemission side, below $E_{\mathrm{F}}$, as the band approaches the Fermi level it appears to dip across into the unoccupied region of the band structure, above the Fermi level, as seen in Figure 6. This occurs at just under $1 / 2$ of the way across the zone and this is supported by the strong rise in the density of states at the Fermi energy at about $k_{\|}=0.5$ $\AA^{-1}$ as plotted in Figure 7. The emergence of the band above $E_{\mathrm{F}}$, as seen with inverse photoemission, confirms the Fermi level crossing by the surface state or surface

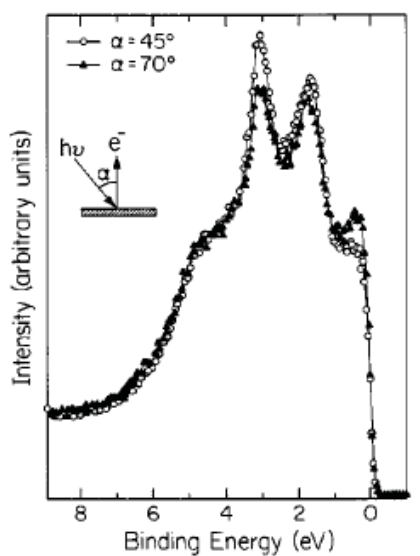

Fig. 8. The light incidence angle dependence of the photoemission energy distribution curves for normal emission $(\bar{\Gamma})$ of $\mathrm{Mo}(112)$, the photoemission spectra are taken at normal emission for $s+p$ polarized light (light incidence angle of 45 degrees) and $p$-polarized light (light incidence angle of 70 degrees). The photon energy is $55 \mathrm{eV}$.

resonance about $1 / 3$ of the way across the zone from $\bar{\Gamma}$ to $\bar{X}$. The fact that the band dispersion obtained from photoemission does not match, precisely, the band dispersion obtained from inverse photoemission at the Fermi level crossing, is a consequence of the finite resolution of both spectroscopies (150 meV and $420 \mathrm{meV}$ ) respectively. It is the combination of photoemission and inverse photoemission that permits the more accurate assessment of the Fermi level crossing. The Fermi level crossing, in theory, is at about 0.45 along $\bar{\Gamma}$ to $\bar{X}[16,17]$, while in the combined experiment it is seen to be at $0.45 \pm 0.03$ $\bar{\Gamma}$ to $\bar{X}$, as indicated in Figures 6 and 7. Because of the photon energy dependence, we have no compelling evidence of surface character for the surface resonance band at binding energies away from the Fermi level, though theory $[16,17]$ does suggest surface weight for this band all along $\bar{\Gamma}$ to $0.45(\bar{\Gamma}-\bar{X})$, as indicated in Figure 6 .

The symmetries of the surface resonances, at normal emission $(\bar{\Gamma})$, can be assigned on the basis of the light incidence angle dependence of the photoemission spectra, shown in Figure 8. The surface resonance at approximately 3.1 and bulk band at $2.4 \mathrm{eV}$ are enhanced with a light incidence angle of 45 degrees and suppressed with a light incidence angle of 70 degrees. For the band with about $1 \mathrm{eV}$ binding energy at $\bar{\Gamma}$ (dispersing towards the Fermi level at $0.45(\bar{\Gamma}-\bar{X}))$, the intensity is enhance with light at 70 degrees light incidence angle. Applying Fermi's golden rule, the symmetry of the bands can be assigned using:

$$
\left(\frac{\mathrm{d} \sigma}{\mathrm{d} \Omega}\right)_{\mathrm{PES}} \propto\left|\left\langle\psi_{\mathrm{f}}|\mathbf{p} \cdot \mathbf{A}| \psi_{\mathrm{i}}\right\rangle\right|^{2} \delta\left(E_{\mathrm{f}}-E_{\mathrm{i}}-h \nu\right)
$$

since the light from the synchrotron is highly plane polarized. The more normal the light incidence angle, the more $s$-polarization and the more vector potential $\mathbf{A}$ of the incident light parallel to the surface. Since, at $\bar{\Gamma}$, the 


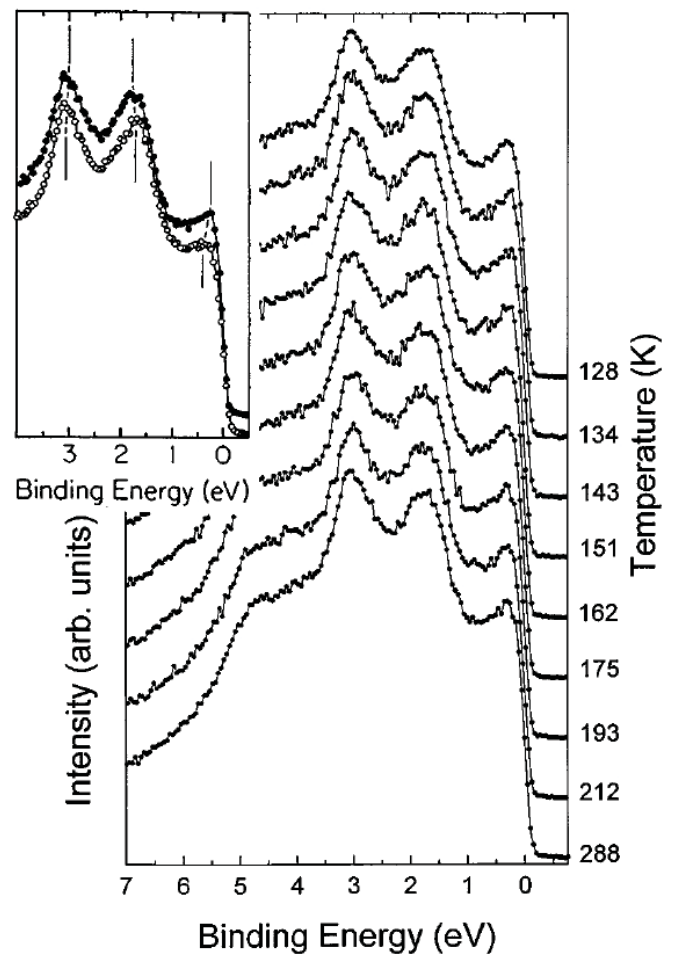

Fig. 9. Normal emission valence band photoemission spectra for $\mathrm{Mo}(112)$ at various temperatures. The light incidence angle is $45^{\circ}(\mathrm{s}+$ $p$ polarized light) and $h v=55 \mathrm{eV}$. The inset highlights the binding energy shifts between $300 \mathrm{~K}(\bullet)$ and $150 \mathrm{~K}(\circ)$ in the occupied bands.

point group symmetry is $C_{2 v}$ the bands observed in photoemission must be $a_{1}\left(s, p_{z}, d_{3 z^{2}-r^{2}}\right), b_{1}\left(p_{x}, d_{x z}\right)$ or $b_{2}\left(p_{y}\right.$, $\left.d_{y z}\right)$. The enhancement of the approximately $3.1 \mathrm{eV}$ surface resonance in more $s$-polarized light indicates that these bands are $b_{1}$ or $b_{2}$ symmetry. The enhancement of the bands near $E_{\mathrm{F}}$ with increasing vector potential along the surface normal (greater light incidence angles) indicates that these bands are $a_{1}$ symmetry in character.

\section{Temperature dependence}

An electronically driven surface reconstruction involves a surface state or resonance crossing the Fermi level [18, 26-36] such as the one(s) just detailed for Mo(112) above. As noted later, for a surface reconstruction, the only bands of importance are those with surface weight that are either very close to the Fermi level or cross the Fermi level $[2,4,5,8]$. Ideally, when a reconstruction occurs, a gap opening occurs near the Fermi energy in the critical directions in $\mathrm{k}$-space. If the phonon modes lock into the lattice at $q=2 \mathbf{k}_{\mathrm{F}}$, where $\mathbf{k}_{\mathrm{F}}$ is the Fermi wave vector, then there is a resulting periodic lattice distortion and the electron density near $E_{\mathrm{F}}$ forms a standing charge density wave [3-5, 33-36]. Bands with considerable surface weight, near the Fermi energy, are implicated in the surface reconstruction of $\operatorname{Mo}(100)[1,6,7]$ and $\mathrm{W}(001)$ [8], indeed half-filled surface states have

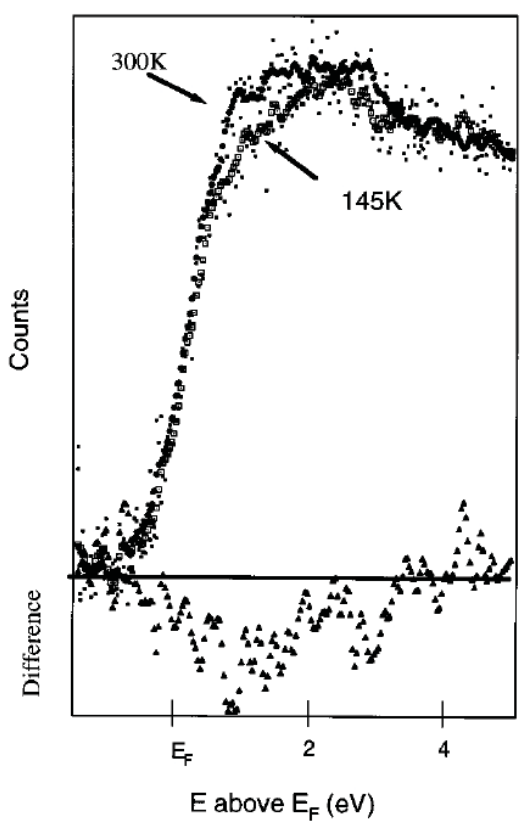

Fig. 10. Temperature dependent inverse photoemission study of Mo(112).

long been held to be responsible for the reconstruction of both $\mathrm{Mo}(100)$ and $\mathrm{W}(100)[1-3,7,8,26-33]$. This is very similar to the observed situation with $\mathrm{Mo}(112)$.

We have just established the presence of such a state along the $\langle\overline{1} \overline{1} 1\rangle$ direction, from $\bar{\Gamma}$ to $\bar{X}$ (where the atomic separation along the rows is $2.73 \AA$ ) where, for the two states crossing the Fermi level, one state is more bulklike and the other more surface sensitive. For Mo(112), the number of possible configurations for the surface reconstruction is increased by the shallow dispersion of the surface resonance/surface state band (near $E_{\mathrm{F}}$ ). Different reconstructions will have accompanying variations of the energy gap at the reconstructed surface Brillouin zone edge. The shallow band dispersion near the surface Brillouin zone midpoint must be a critical factor in driving $\operatorname{Mo}(112)$ to a $(1 \times 2)$ reconstruction with the adsorption of hydrogen [11]. Based on the $\mathrm{Mo}(112)$ band structure, the Fermi level crossing is between $1 / 2$ and $1 / 3$ from $\bar{\Gamma}$ to $\bar{X}$. Thus both $(3 \times b),(6 \times b),(9 \times b), \ldots(3 m \times b)($ where $m$ and $b$ are integers) reconstructions, as discussed below, and the $2 \times 1$ reconstruction [11], are therefore favored. The dispersion from $\bar{\Gamma}$ to $\bar{Y}$, on the other hand, suggests reconstructions of the form $(a \times 3),(a \times 6),(a \times 9), \ldots(a$ $\times 3 n)$ (where $a$ and $n$ are integers), so that more complex reconstructions of the form $(3 n \times 3 m)$ are possible.

Some indications for a temperature dependent reconstruction are evident in both the temperature dependence of the photoemission spectra (Fig. 9) and inverse photoemission spectra (Fig. 10). As seen in Figure 9, a decrease in temperature below $300 \mathrm{~K}$ leads to little shift in photoemission binding energies except near the Fermi level. There is a shift or decrease in the density of states in both the oc- 


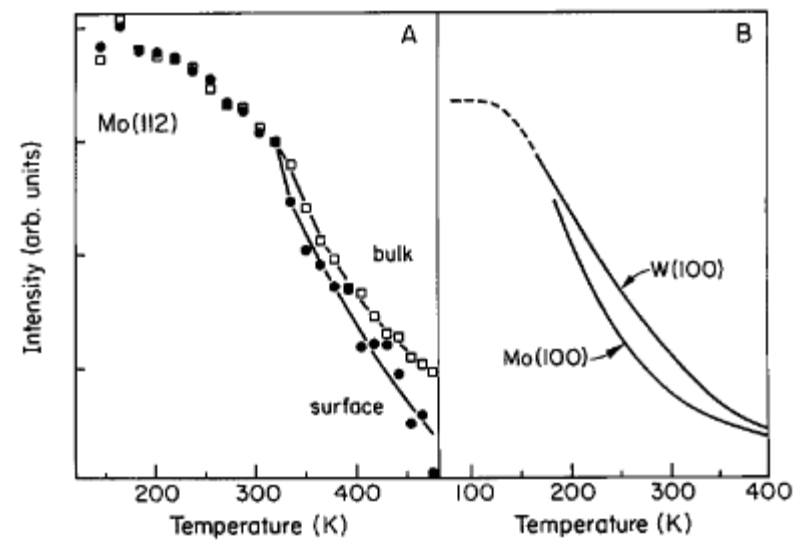

Fig. 11. (A) The photoemission peak intensities of the $2.8 \mathrm{eV}$ (surface) feature and $1.7 \mathrm{eV}$ (bulk) feature (see Fig. 2) as a function of temperature. (B) LEED diffraction spot intensities as a function of temperature for the (100) surface of both molybdenum and tungsten, taken from reference [3].

cupied (Fig. 9 insert) and the unoccupied band structure at zone center $(\bar{\Gamma})$, with decreasing temperature, as seen in Figure 10. We anticipate from theory [16] some oscillator strength from surface resonances at zone center $(\bar{\Gamma})$, though small shifts in intensity from a change in the surface screening parameter (such as a changes photoemission intensity from plasmon or surface plasmon resonances) cannot be excluded. Changes near the Fermi level, at zone center $(\bar{\Gamma})$, suggest a gap opening and a binding energy shift of the surface resonances away from the Fermi level. This is consistent with an electronic or surface reconstruction whose onset is well below room temperature [4].

Temperature is observed to affect both the binding energies and intensity in photoemission and inverse photoemission. While intensity variations, with temperature, in electron spectroscopies, are expected because of the Debye scattering contributions, such dynamical scattering cannot be the sole cause of the temperature variations observed. Deviations from expected temperature dependent dynamical scattering are observed.

At higher temperatures, well above the temperature where the decrease in density of states near the Fermi level is observed, photoemission intensities follow the expected temperature dependence of electron-phonon scattering (Debye scattering), as previously noted [18]. (For our purposes we only need to consider the effective (as noted by Waldfried et al. [18]), not the true surface Debye temperature.) For temperatures less than $250 \mathrm{~K}$, as summarized in Figure 11, deviations from the expected Debye scattering occur. The surface electronic structure is much more sensitive to temperature variations than the neighboring bulk band features at $4 \mathrm{eV}$ and $1.7 \mathrm{eV}$. Not surprisingly, the deviation from expected dynamical scattering temperature dependence is most apparent in the surface resonances of Figure 9. We have plotted the intensity of the surface resonance, in the photoemission spectra at 3.1 to $2.8 \mathrm{eV}$ bind- ing energy, where the surface resonance intensity is most easily abstracted in Figure 11, rather than the states near the Fermi energy where the signal to noise is not as large. This variation in photoemission intensities cannot be modeled by simple dynamic motion variations in temperature [18].

This temperature dependent intensity variation is not the first such evidence of a surface reconstruction. The photoemission or diffraction intensity as an indicator of a surface reconstruction was first observed on the (100) surface of both molybdenum and tungsten by Felter et al. [3]. For $\mathrm{Mo}(100)$ and $\mathrm{W}(100)$, a temperature dependent reconstruction to $c(2 \times 2)$ was observed in low-energy electron diffraction (LEED) patterns. As seen in Figure 8, our photoemission results for the Mo(112) surface are similar to deviations from the expected Debye related temperature dependence of the LEED intensity for the W(100) and Mo(100) surfaces.

Careful, high resolution photoemission measurements near the Fermi level crossings, are now possible on some photoemission spectrometer-beamline combinations. Such measurements would provide even better indications of the temperature dependent changes to the band structure than the results presented here.

\section{Adsorbate induced reconstruction}

Not surprising, given the experience with $\mathrm{Mo}(100)$ and $\mathrm{W}(100)$, surface reconstructions of $\mathrm{Mo}(112)$ are also initiated by small amounts of adsorbates, such as oxygen or carbon. Characteristic of a surface state or surface resonance, the density of states near the Fermi energy is altered with small amounts of an adsorbate. This density of state in inverse photoemission gradually disappears with increasing amounts of oxygen in agreement with other studies of this system [18] as seen in Figure 12. This is more evident in the difference curve in that same figure where the clean Mo(112) spectrum has been subtracted from the oxygenated surface spectra. This influence upon the Fermi level density of state can help drive a surface reconstruction. Unfortunately, midzone along $\bar{\Gamma}$ to $\bar{X}$, the $C_{1 h}$ symmetry permits O $2 p_{x}$ hybridization with Mo $4 d_{3 z^{2}-r^{2}}$. Only at zone center $(\bar{\Gamma})$ is such hybridization forbidden by symmetry $\left(C_{2 v}\right)$. The change in the density of states at $E_{\mathrm{F}}$ alone is not compelling evidence of a reconstruction.

Figure 13 provides examples of changes in the surface unit cell for the $\operatorname{Mo}(112)$ surface with oxygen. LEED patterns with different oxygen exposures are shown and the change in structure that occurs with increasing adsorbate is clear. The amount of oxygen is quite small, only 0.15 Langmuir $\left(1\right.$ Langmuir $=1 \times 10^{-}$ ${ }^{6}$ torr-seconds), are necessary to drive the surface into the first structure. To establish that the LEED patterns are reconstructions and not just simply due to a ordered 


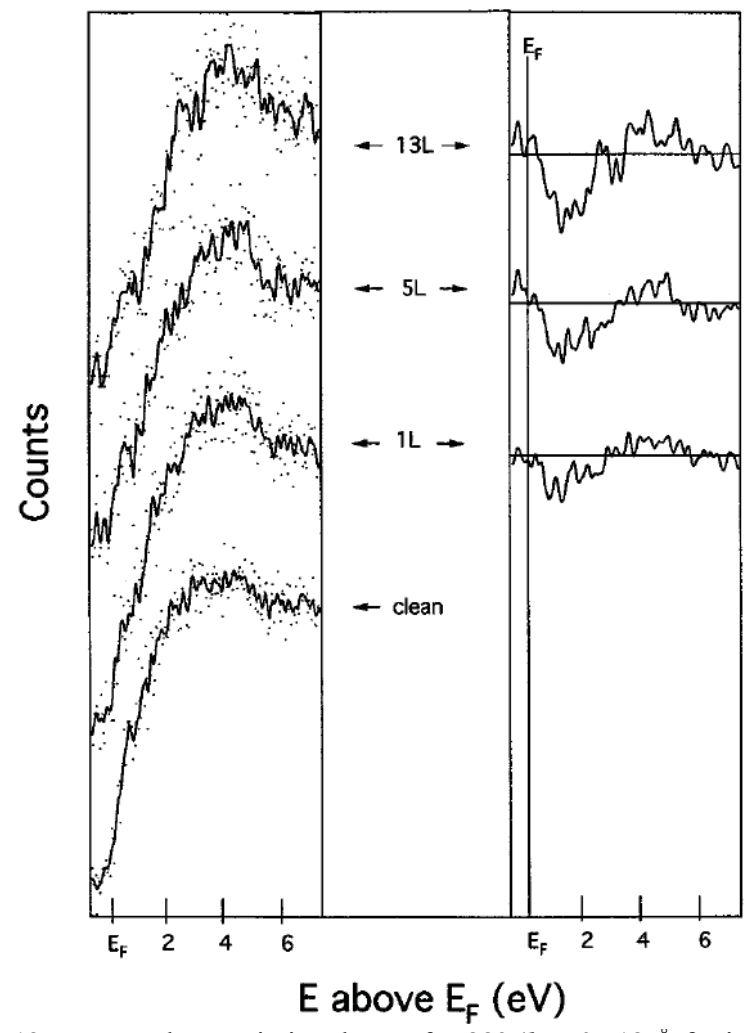

Fig. 12. Inverse photoemission data at $\theta=22^{\circ}\left(k_{\|}=0.512 \AA\right.$ for increasing oxygen exposure. The difference curve was obtained by subtracting the clean $\mathrm{Mo}(112)$ spectrum from the oxygenated spectra. This is the position of the Fermi level crossing for clean $\mathrm{Mo}(112)$ along $\bar{\Gamma}$ to $\bar{X}$.

overlayer structure, complementary scanning tunneling microscopy measurements were undertaken.

The various reconstructed surface structures have been identified with STM as a function of increasing surface carbon contamination. Figure 14 shows the unreconstructed Mo(112) surface (image (a)) and the adsorbate induced reconstructions (panels (b)-(c)). The unreconstructed Mo(112) surface shows the characteristic "row" pattern, due to the rectangular surface unit cell with dimensions of $4.45 \AA$ perpendicular to the rows and $2.73 \AA$ along the rows. With small amounts of adsorbates the surface reconstructs, forming a "checkers board-like" missing row structure (Fig. 14b). The image shows a missing row with every two existing rows and changing brightness along the rows with a periodicity of 9 unit cells, consistent with the $(9 \times 3)$ LEED pattern, that is observed for the initial reconstruction. A "buckling" of the surface atoms along the rows is also possible. Additional adsorption of carbon changes the surface structure further through a coexisting reconstructed arrangement (Fig. $14 \mathrm{c})$ to the $(6 \times 12)$ reconstruction (Fig. 14d). This reconstruction is characterized by long (many $1000 \AA$ in length) 5 unit cell wide "stripes," separated by a missing row. Along these lines there is a periodic variation in electron charge density, indicated by the varying tunnel current intensity. This "stripe"-like structure is consistent with the $(6 \times 12)$ LEED pattern.

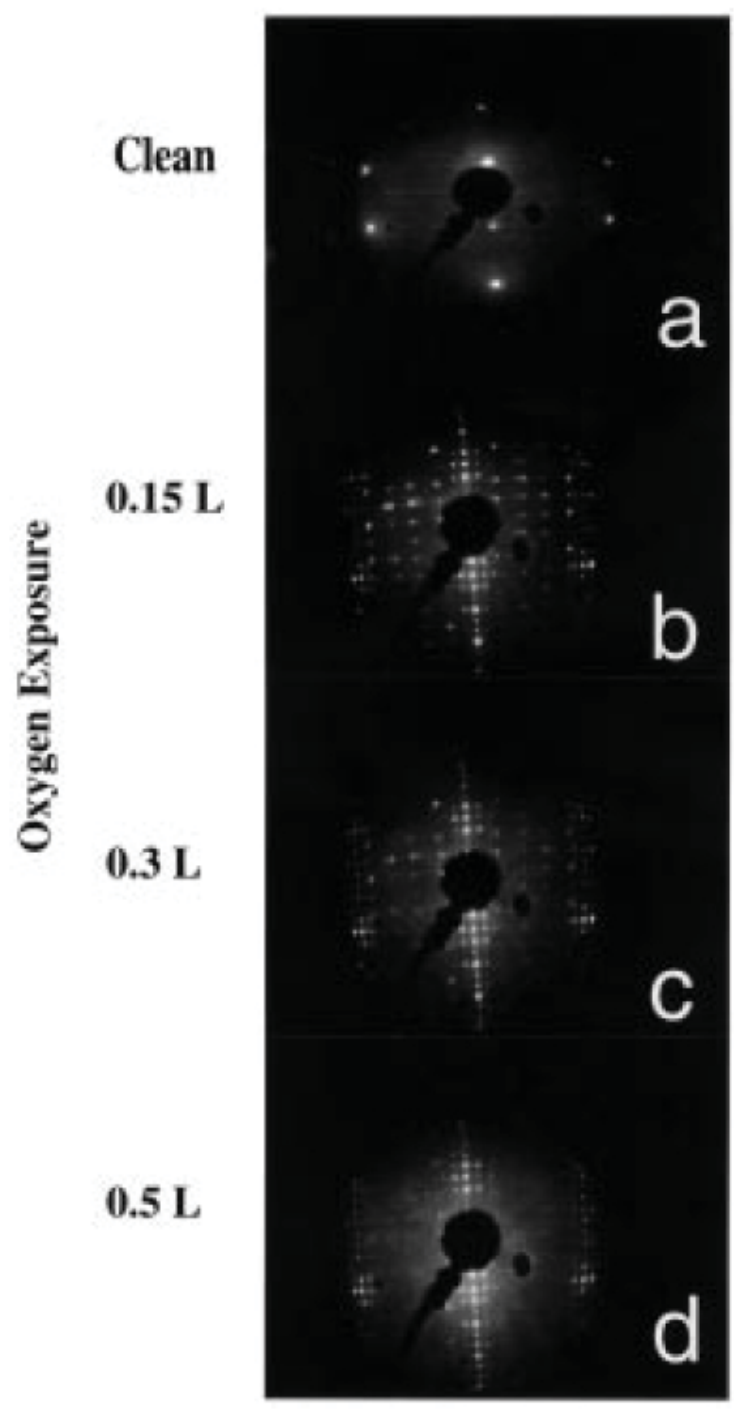

Fig. 13. Mo(112) LEED (beam voltage $=73.3 \mathrm{eV}$ ) patterns with increasing oxygenation. The clean $(1 \times 1)$ structure $(a)$ reconstructs to a $(3 \times 9)$ structure $(b)$, to coexisting structures $(c)$ and to a $(6 \times 12)$ structure (d) with a small amount of oxygen $(0.5 \mathrm{~L})$.

It is clear that the morphological changes in the surface, observed by STM, are far too large to be accounted for by just a small amount of contamination, thus both the LEED and the STM observations are indicative of adsorbate induced reconstructions, not the adsorbate overlayer structure per se. The structural results of the surface reconstructions obtained by STM and LEED are in excellent agreement with each other and both are consistent with the molybdenum band structure from which we can predict that $(3 m \times 3 n)$ reconstructions are favored. With minimal surface oxygen (or carbon) coverages the clean $(1 \times 1)$ structure completely reconstructs to a $(9 \times 3)$, a $(6$ $\times 12$ ), and a coexisting structure. The STM images show a reconstruction similar to that observed in the surface of a number of transition metal carbides.

Similar changes to electronic structure are implicated in the adsorbate induced reconstructions of W(111) 

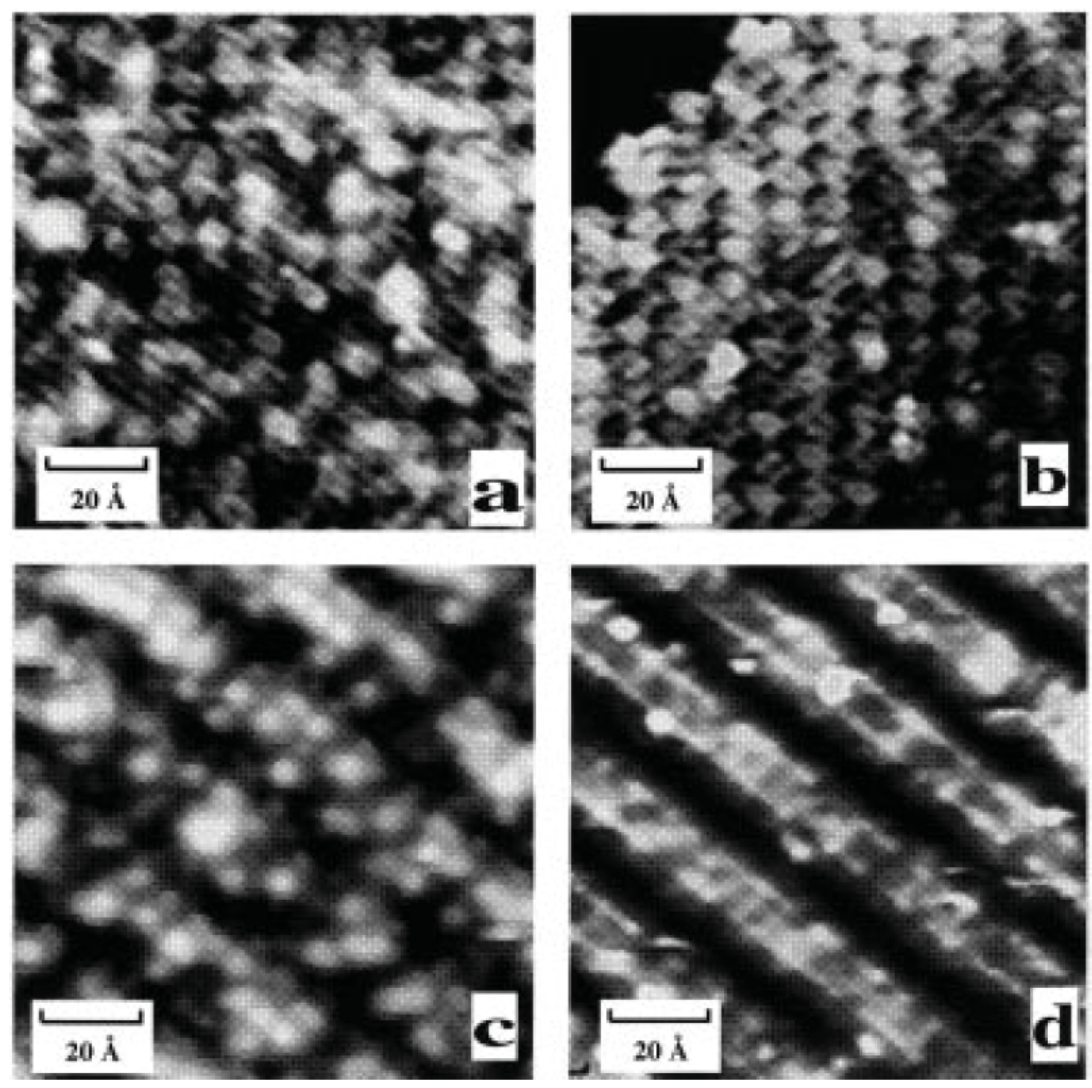

Fig. 14. Mo(112) STM images (constant current mode) with increasing carbonation. The clean $(1 \times 1)$ "line" structure $(a)$ reconstructs to a $(3 \times$ 9) "checker board" structure (b), to coexisting structures (c), and to a $(6 \times 12)$ "stripe" structure (d) with small amount of carbon.

$[15,37], \mathrm{W}(112)$ [38-40], $\mathrm{Mo}(100)[7,41,42], \mathrm{Mo}(111)$ $[43,44], \operatorname{Mo}(110)$ [45], and W(110) [46]. Adsorbate induced surface reconstructions are a common phenomena and have been seen earlier for $\operatorname{Mo}(112)[11,12]$ as well as on the (100) surface of both molybdenum and tungsten [3, 7, 47] where Cs [48], Sn [49], S [50-52], CO [53], $\mathrm{N} \mathrm{[54]} \mathrm{are} \mathrm{all} \mathrm{found} \mathrm{to} \mathrm{induce} \mathrm{reconstructions} \mathrm{on}$ the Mo(100) surface. Further examples are also found on the (111) surface of both molybdenum and tungsten [1-14]. In particular, Pd and Pt are seen to reconstruct $\mathrm{W}(111)$ and $\mathrm{Mo}(111)$ to (112) facets $[13,43]$. In their LEED patterns, Guan et al. [43] observed a $(1 \times n)$ superstructure on the (112) facets induced in the reconstructions of $\mathrm{W}(111)$ and $\mathrm{Mo}(111)$ with a critical wave vector close to that which we observe. Bode and coworkers have observed reconstructions of W(110) induced by carbon with scanning tunneling microscopy [46]. In addition, Terrasi et al. observed a narrow gap and no Fermi level crossing for the quasi-two-dimensional material $\mathrm{Mo}_{4} \mathrm{O}_{11}$, consistent with our evidence of the effects of oxygen on molybdenum [55]. These results support the postulate that exposure of $\mathrm{Mo}(112)$ to oxygen or carbon results in a small gap opening at the Fermi level.

\section{Summary}

We have experimentally mapped out the Mo(112) band structure using photoemission and inverse photoemission. Evidence for a surface reconstruction induced by temperature and adsorbates are presented, associated with the change of the surface electronic structure. We postulate that the transition at or about $250 \mathrm{~K}$ is electronically driven by a Fermi level crossing of a surface resonance. As such, this behavior is very similar to earlier work with $\mathrm{Mo}(100)$ [6-9] and the recent study of $\mathrm{W}(100)$ [10]. The temperature dependence of the band structure for the (112) surface [3], is similar to changes induced by exposure to an adsorbate, either a main group element, as noted here, or hydrogen as noted elsewhere [11]. Adsorbates are seen to have a dramatic affect upon the surface structure.

\section{Note added in proof}

Further evidence of lateral displacements, possibly leading to a temperature dependent reconstruction of the Mo(112) surface have recently been found from detailed 
LEED studies (D. Kolthoff, H. Pfnur, A.G. Fedorus, V. Koval, A.G. Naumovets, Surf. Sci. 439, 224 (1999)). This provides some additional support to the results presented here.

This work was funded by the NSF through grants DMR9802126 and INT-9300238. We would like to thank G. Katrich who helped initiate this study. A significant portion of this work was carried out at the Synchrotron Radiation Center in Stoughton, Wisconsin, which is supported by the NSF.

\section{References}

1. J.W. Chung, Surface states and reconstruction of the Mo(001) surface, in Electronic Surface and Interface States on Metallic Systems, edited by E. Bertel, M. Donath (World Scientific, Singapore, 1995), Vol. 67.

2. E. Tosatti, Surface States, Surface Metal-Insulator, and Surface Insulator-Metal Transitions, in Electronic Surface and Interface States on Metallic Systems, edited by E. Bertel, M. Donath (World Scientific, Singapore, 1995), Vol. 67.

3. T.E. Felter, R.A. Barker, P.J. Estrup, Phys. Rev. Lett. 38, 1138 (1977).

4. A. Fasolino, E. Tosatti, Phys. Rev. B 35, 4264 (1987).

5. A. Fasolino, G. Santoro, E. Tosatti, Surf. Sci. 125, 317 (1983).

6. K. Smith, S. Kevan, Phys. Rev. B 43, 3986 (1991).

7. K. Smith, S. Kevan, Phys. Rev. B 45, 13642 (1992).

8. J.W. Chung, K.S. Shin, S.C. Hong, Mod. Phys. Rev. B 21, 865 (1993); J.C. Campuzano, J.E. Ingesfield, D.A. King, C. Somerton, J. Phys. C 14, 3099 (1988); K.E. Smith, G.S. Elliot, S.D. Kevan, Phys. Rev. B 42, 5385 (1990); H.K.S. Shin, H.W. Kim, J.W. Chung, Surf. Sci. 385, L978 (1997).

9. A.G. Naumovets, Sov. Sci. Rev. Phys. A 5, 443 (1984).

10. V.K. Medvedev, I.N. Yakovkin, Sov. Phys. Solid State 21, 187 (1979).

11. G.P. Lopinski, J.A. Prybyla, P.J. Estrup, Surf. Sci. 296, 9 (1993).

12. G.P. Lopinski, J.A. Prybyla, P.J. Estrup, Surf. Sci. 315, 269 (1994).

13. T.E. Madey, J. Guan, S.H. Nien, C.Z. Dong, H.S. Tao, R.A. Campbell, Surf. Rev. Lett. 3, 1215 (1996).

14. J. Guan, R.A. Campbell, T.E. Madey, Surf. Sci. 341, 311 (1995).

15. H.S. Tao, C.H. Nien, T.E. Madey, J.E. Rowe, G.K. Wertheim, Surf. Sci. 55, 357 (1996).

16. I.N. Yakovkin, Surf. Sci. 389, 48 (1997).

17. I. N. Yakovokin, G.A. Katrich, A.T. Lobrets, Yu.S. Vedula, A.G. Naumovets, Prog. Surf. Sci. 59, 355 (1998).

18. C. Waldfried, D.N. McIlroy, Jiandi Zhang, P.A. Dowben, G.A. Katrich, E.W. Plummer, Surf. Sci. 363, 296 (1996).

19. S.L. Weng, E.W. Plummer, T. Gustafsson, Phys. Rev. B 18, 1718 (1978).

20. G.P. Kerker, K.M. Ho, M.L. Cohen, Phys. Rev. B 18, 5473 (1978); R.C. Cinti, E. Al Khoury, B.K. Krakaverty, Phys. Rev. B 14, 3296 (1976).

21. C. Noguera, D. Spanjaard, D.W. Jepsen, Phys. Rev. B 17, 607 (1978).

22. P. Soukasian, R. Rivan, J. Lecante, E. Wimmer, S.R. Chubb, A.J. Freeman, Phys. Rev. B 31, 4911 (1985); S.R. Chubb, E. Wim- mer, A.J. Freeman, J.R. Hiskes, A.M. Karo, Phys. Rev. B 36, 4112 (1987).

23. P.W. Erdman, E.C. Zipf, Rev. Sci. Instrum. 53, 225 (1982).

24. C. Waldfried, T. McAvoy, D. Welipitiya, Takashi Komesu, P.A. Dowben, E. Vescovo, Phys. Rev. B 58, 7434 (1998).

25. P.A. Dowben, D. LaGraffe, M. Onellion, J. Phys. Condens. Matt. 1, 6571 (1989).

26. J.E. Inglesfield, J. Phys. C 11, L69 (1978); 12, 149 (1979).

27. M. Posternak, H. Krakauer, A.J. Freeman, D.D. Koelling, Phys. Rev. B 21, 5601 (1980); E. Wimmer, H. Krakauer, M. Weinert, A.J. Freeman, Phys. Rev. B 24, 864 (1981); M. Posternak, H. Krakauer, A.J. Freeman, D.D. Koelling, Phys. Rev. B 30, 4828 (1984).

28. C.L. Fu, S.O. Ohnishi, H.J.F. Jansen, A.J. Freeman, Phys. Rev. B 31, 1168 (1985).

29. K. Terakura, I. Terakura, Y. Teraoka, Surf. Sci. 86, 535 (1979); K. Terakura, I. Terakura, N. Hamada, Surf. Sci. 103, 103 (1981).

30. H. Krakauer, Phys. Rev. B 30, 6834 (1984); D. Singe, Krakauer, Phys. Rev. B 37, 3999 (1988).

31. G. Treglia, M.C. Desjouqueres, D. Spanjaard, J. Phys. C 16, 2407 (1983).

32. C.L. Fu, A.J. Freeman, E. Wimmer, M. Weinert, Phys. Rev. Lett. 54, 2261 (1985).

33. H. Krakauer, M. Posternak, A.J. Freeman, Phys. Rev. Lett. 43, 1885 (1979).

34. J.W. Chung, K.S. Shin, D.H. Baek, C.Y. Kim, H.W. Kim, S.K. Lee, C.Y. Park, S.C. Hong, T. Kinoshida, M. Watanabe, A. Kakizaki, T. Ishi, Phys. Rev. Lett. 69, 2228 (1992).

35. E. Tosatti, Solid State Commun. 25, 637 (1978).

36. J.C. Campuzano, D.A. King, C. Somerton, J.E. Inglesfield, Phys. Rev. Lett. 45, 1649 (1980)

37. N.J. Taylor, Surf. Sci. 2, 544 (1964).

38. B.J. Hopkins, G.D. Watts, Surf. Sci. 44, 237 (1974).

39. J.B. Benziger, R.E. Preston, Surf. Sci. 151, 183 (1985).

40. J.M. Chen, C.A. Papageorgopoulos, Surf. Sci. 21, 377 (1970).

41. H.M. Kennett, A.E. Lee, Surf. Sci. 48, 606 (1975).

42. H.M. Kennett, A.E. Lee, Surf. Sci. 48, 633 (1975).

43. J. Guan, R.A. Campbell, T.E. Madey, J. Vac. Sci. Tech. 13, 1484 (1995).

44. C. Zhang, M.A. VanHove, G.A. Somorjai, Surf. Sci. 149, 326 (1985).

45. H.M. Kennett, A.E. Lee, Surf. Sci. 48, 591 (1975).

46. M. Bode, R. Pascal, R. Wiesendanger, Surf. Sci. 344, 185 (1995).

47. H.K.A. Kan, S. Feuerstein, J. Chem. Phys. 50, 3618 (1969).

48. R. Riwan, P. Soukiassian, S. Zuber, J. Cousty, Surf. Sci. 146, 382 (1984).

49. O. Nishikawa, M. Wada, M. Konishi, Surf. Sci. 97,16 (1980).

50. V. Maurice, L. Peralta, Y. Berthier, J. Oudar, Surf. Sci. 148, 623 (1984).

51. M. Salmeron, G.A. Somorjai, Surf. Sci. 126, 410 (1983).

52. D. Tabor, J.M. Wilson, J. Crys. Gro. 9, 60 (1971).

53. L.J. Clarke, J. Vac. Sci. Tech. 16, 651 (1979).

54. A. Ignatiev, F. Jona, D.W. Jepsen, P.M. Marcus, Surf. Sci. 49, 189 (1975).

55. A. Terrasi, M. Marsi, H. Berger, F. Gauthier, L. Forro, G. Margaritondo, R.J. Kelley, M. Onellion, Z. Phys. B. 100, 493 (1996). 